\title{
A rare clinical presentation of urinary bladder carcinoma: A case report
}

\author{
Rekha N Patil ${ }^{1, *}$, Sandhya Poflee ${ }^{2}$, Waman Raut ${ }^{3}$ \\ ${ }^{\mathbf{1}}$ Assistant Professor, ${ }^{2}$ Associate Professor, ${ }^{3}$ Professor and Head, Dept. of Pathology, GMC, Nagpur, Maharashtra, India
}

*Corresponding Author:

Email: rekhapatil3454@yahoo.in

Received: $04^{\text {th }}$ October, 2017

Accepted: $08^{\text {th }}$ December, 2017

\begin{abstract}
Metastasis of carcinoma to regional lymph nodes is of common occurrence. Cervical lymph nodes are the commonest lymph nodes to be sampled for detection of metastasis from head neck and upper are digestive tract malignancies. Morphological confirmation of metastasis in the lymph nodes either by cytology or histopathology decides the further plan of treatment for the patient. Rarely cervical lymph nodes can show evidence of metastasis from urinary bladder carcinoma. In the present case the cervical lymph node biopsy was helpful in the diagnosis and treatment of the patient. The histopathological findings were later confirmed by immunohistochemistry supporting the diagnosis of metastasis of urinary bladder carcinoma to the left cervical lymph nodes. To arrive at the correct diagnosis complete clinical workup, clinicoradiological correlation and high index of suspicion is required. Reporting this case will emphasize the rare clinical presentation of urinary bladder carcinoma.
\end{abstract}

Keywords: Urinary bladder carcinoma, Cervical lymph nodes, Metastasis, Uroplakin.

\section{Introduction}

Cancers arising from the head neck region and upper areodigestive tract frequently metastasise to the cervical lymph nodes. Cervical lymph node metastasis can seldom be seen in cancers arising from sites outside the head, neck region and from organs below the diaphragm. ${ }^{1-3}$

Urinary bladder carcinoma (UBC) usually metastasise to the regional lymph nodes, lungs, bone, adrenal glands, pleura, peritoneum. Rare cases of UBC metastasizing to brain, intestine, choroid and skin are also reported..$^{2-7}$

UBC metastasizing to cervical group of lymph nodes is rare. ${ }^{1-4}$

We present a case of cervical lymph node metastasis of UBC. The computed xcvtomography (CT) of the patient showed mild urinary bladder wall thickening with a small polypoidal mass lesion at the right vesicouerteric junction. His cervical lymph node mass was biopsied. The biopsy showed metastasis of UBC. It was uroplakin positive, confirming the

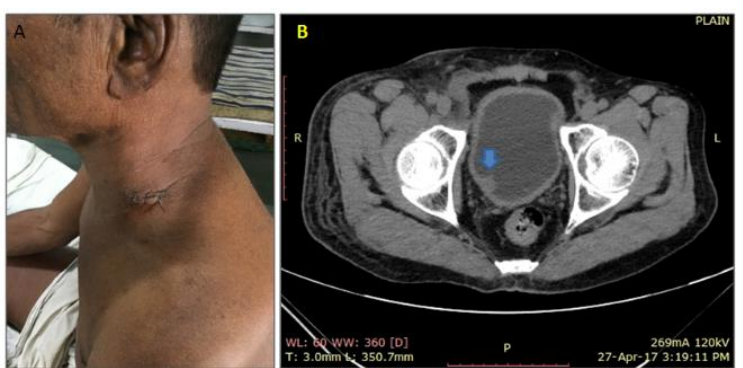

Fig. 1:

He had haemoglobin of $8 \mathrm{gm} / \mathrm{dl}$ with normal total and differential leukocyte count. Urine microscopic diagnosis of metastasis of UBC to the left cervical lymph node.

This case is presented to emphasise the rare clinical presentation of UBC.

\section{Case Report}

A 65 years male patient came with the complaints of pain and swelling of the right thigh since 15 days. The swelling began at the hip joint level and gradually progressed downwards. He also complained of a swelling in the left cervical region. He had no urinary complaints, no history of trauma and haematuria.

His vital parameters were stable. He had nonpitting oedema of right supra-pubic and right upper thigh region. His oral, nasal examination did not reveal any lesion. His respiratory, cardiovascular and central nervous system examination was within normal limit. Abdomen was soft with no palpable lump.

$\mathrm{He}$ had a $2 \times 2 \mathrm{~cm}$ swelling in the left cervical region. It was firm to hard, fixed to the underlying structures with normal overlying skin. [Fig. 1]

examination showed no aberrant cells and red blood corpuscles (RBC).

His HIV status was non- reactive. HBsAg was negative. His urea, creatinine and electrolytes were normal.

Computed Tomography (CT) of abdominopelvic region showed a $1.5 \times 1.5 \mathrm{~cm}$ polypoidal lesion at the right vesicoureteric junction. [Fig. 1] The thickness of urinary bladder wall was slightly increased $(8 \mathrm{~mm})$. There was mild dilation of the right ureter. There were multiple enlarged necrotic lymph nodes along the right iliac vessels. Enlarged lymph nodes were also seen in the pre and para aortic, aortocaval region, right renal hilar and right inguinal region. The largest was of size 
$3 \times 2.4 \mathrm{~cm}$. There was thrombosis of external iliac vessels along with encasement by the external iliac lymph nodes. There was a lobulated necrotic lesion of size $1.7 \times 2.1 \times 2.9 \mathrm{~cm}$ at the lateral border of right psoas muscle. The visualised lower lung field showed a few random nodules along with thickening of the horizontal fissure which were suggestive of metastasis in the lungs.

The biopsy of the left cervical lymph node was done and we received the lymph node of $1.5 \mathrm{x} 1.5 \mathrm{~cm}$. The sections showed the lymph node structure replaced by cells arranged in sheets, trabeculae and at places papillary pattern. The cells had moderate amount of eosinophilic cytoplasm and round hyperchromatic nuclei. There were occasional mitosis. There were no areas of necrosis and nuclear pleomorphism. On immunohistochemistry the cells showed cytoplasmic positivity for uroplakin III [Fig. 2]. After the clinicoradiological correlation the diagnosis of metastasis of transitional cell carcinoma of urinary bladder was offered.

Patient was not willing for cystoscopy and was discharged against the medical advise.

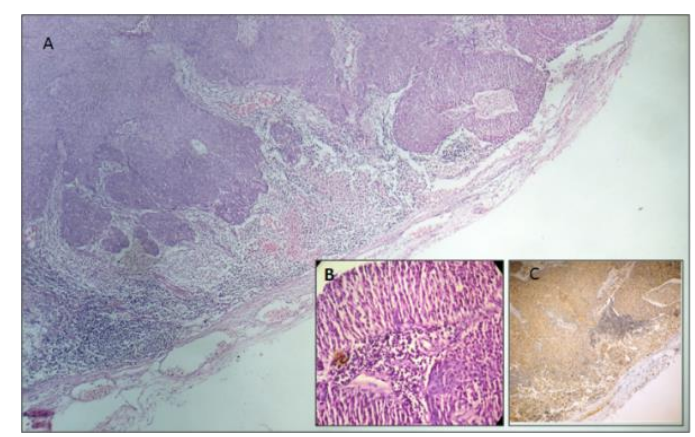

Fig. 2:

\section{Discussion}

The primary lymphatic drainage of the urinary bladder is into the external, internal iliac and obturator group of lymph nodes. These groups of lymph nodes are involved in about $20-40 \%$ cases of urinary bladder carcinoma (UBC). The involvement of common iliac group of lymph nodes can occurs as a secondary drainage. ${ }^{3,4,8}$

It is extremely rare for the UBC to metastasis to the cervical lymph nodes. Cervical lymph node metastasis in patients with UBC indicates a widespread disease and a poor

\section{Prognosis. ${ }^{1-4,8}$}

In a large study of 845 patients of urogenital tract tumors, only $31(3.7 \%)$ patients showed metastatic deposits in the head and neck region. These tumors included all cases of kidney, prostate, bladder, testis, penis, urethra and ureter. ${ }^{2,3}$
The spread to the head and neck region occurs through the hematogenous route (vertebral veins) and also by lymphatics. ${ }^{2-4,8}$

In 1 to $8 \%$ patients of UBC metastasis can be seen at unusual sites. This is because the UBC has a varied metastatic potential. ${ }^{4}$

Mutahir A. Tunio et al reported two cases of UBC with metastasis at unusual sites. One was a case of choriodal metastasis in a 64 years male. The other case was of a 58 years male with unilateral left supra clavicular lymph node metastasis. Both cases were of muscle invasive urinary bladder carcinoma and the metastasis developed after the treatment of UBC. ${ }^{3,6}$

In the study by Waihenya and Mungai the most important clinical feature was haematuria present in $98 \%$ patients of UBC. ${ }^{9}$ In the present case there was no haematuria which is a common clinical presentation of UBC.

The initial presenting clinical feature in this case was right lower limb oedema and left cervical lymph node enlargement. A very rare clinical presentation of UBC.

On histopathological examination of lymph nodes it is difficult to differentiate between metastasis of squamous cell carcinoma and urothelial bladder carcinoma. ${ }^{1}$ The presence of right vesicoureteric mass lesion and the urinary bladder wall thickening on CT and Uroplakin positivity helped in making the diagnosis of the present case. The cervical lymph node lesions must be interpretated with complete clinical and radiological workup. Immunohistochemistry uroplakin positivity definitely helps in the diagnosis of these cases.

Head neck and upper aerodigestive tract malignancy commonly metastasis to the cervical lymph nodes..$^{1-3}$

There is a very low incidence of UBC metastasising to the cervical group of lymph nodes and it indicates a poor prognosis and a widespread disease. ${ }^{1-}$ 3

In the study by Babaian 1980 he observed that within a year of primary diagnosis of UBC the metastatic lesion were clinically obvious. ${ }^{7}$

In the present case the cervical metastasis was one of the initial clinical presenting symptoms along with right lower limb oedema.

In the present case the $\mathrm{CT}$ of abdominopelvic region showed the presence of a mass at the vesicoureteric junction with mild thickening of urinary bladder wall. The strong cytoplasmic positivity for uroplakin also helped us in the diagnosis of this case of UBC with cervical metastasis.

Lymphatic filariasis is the most common cause of secondary lymphedema worldwide and in developed countries malignancy is the most prevailing cause. ${ }^{5}$ In the present case the patient presented with oedema of the right lower limb making filariasis as one of the clinical differential diagnosis. 
When the malignant cell infiltrate the superficial and deeper dermal lymphatics and the lymph node, there can be lymphedema. In the present case there was painful and rapid onset lymphedema which can be explained by wide spread intraabdominal and inguinal lymph nodes involvement, however there were no cutaneous lesions seen in the present case. Chin-Pao Chang et al reported the case with cutaneous metastasis in case of UBC. ${ }^{5}$

Uroplakin (UP) is a transmembrane protein. There are numerous studies which investigated the role of uroplakin in the primary and metastatic tumors of UBC. UP shows a membranous and or cytoplasmic dispersal. Uroplakin is an useful immunohistochemistry (IHC) marker to demonstrate the urothelial origin of the tumor. In this case the cells showed cytoplasmic positivity for uroplakin. ${ }^{10}$

In cases of UBC, commenting on the standard treatment of cervical lymph node metastasis, is problematic as these patients are few and also have a short survival. ${ }^{1}$

For effective treatment there should be early detection colposcopy which remains a gold standard for the diagnosis of UBC.

Cervical lymph node metastasis means a widespread disease and palliative treatment is mostly recommended. ${ }^{1-3}$

\section{Conclusion}

Cervical mass in a patient should be interpreted with proper knowledge of complete clinical and radiological findings. Uroplakin positivity can be helpful in arriving at the diagnosis of metastasis of urinary bladder carcinoma.

\section{References}

1. Fernando López, Juan P. Rodrigo, Carl E. Silver, Missak Haigentz Jr, Justin A. Bishop, et al Cervical lymph node metastases from remote primary tumor sites Head Neck . 2016 April;38(Suppl 1): E2374-E2385. doi:10.1002/hed.24344.

2. Ore Ogunyemi A Rojas K Hematpour D Rogers C Head c Bennett. Metastasis of genitourinary tumors to the head and neck region.Eur Arch otorhinolaryngol 2010;267:273-79.

3. Mutahir A Tunio, Mushabbab A 1 Asiri, Yasser Bayoumi, Mohsin Fareed, Shoaib Ahmad. Cervical lymph node metastasis from transitional cell carcinoma of urinary bladder: Case report and review of literature. Journal of Solid Tumors 2012;2(3):59-62.

4. Jae Hoon Chung, Joo Yong Lee, Ju Yeon Pyo, Young Ha Oh, Seung Wook Lee, Hong Sang Moon, Hong Yong Choi. Brain and Skin Metastasis from Urothelial Carcinoma of the bladder. Korean J Urol2013;54:66-68.

5. Chang CP, Lee IY, Shih HJ. Unusual presentation of cutaneous metastasis from bladder urothelial carcinoma. Chin J Cancer Res 2013;25(3):362-365.

6. Tunio M, Rafi M, Hashmi A, Mohsin R, Raza SS. Choroidal metastasis from primary transitional cell carcinoma of urinary bladder: A case report and review of literature. Rawal Med J 2011;36:69-70.

7. Babaian RJ, Johnson DE, Llamas L, Ayala AG. Metastases from transitional cell carcinoma of urinary bladder. Urology 1980;16:142-44.

8. Venkat Pavan Kancharlaa Frederick A. Gulmib Aref Aghelia Michael Degenb Arash Goharic Ming Jiangd J.C. Wanga Transitional Cell Carcinoma of the Bladder Manifestating as Malignant Lymphoma with Generalized Lymphadenopathy Case Rep Oncol 2010;3:125-130.

9. Waihenya CG, Mungai PN. Management of transitional cell carcinoma of the urinary bladder at Kenyatta National Hospital, Nairobi. East Afr Med J 2006;83(12);679-83.

10. Kiara klopfer, Brett Delahunt, Michael Adamson, Hemamali Samaratunga. Value of uroplakin III in distinguishing variants of primary bladder urothelial carcinoma from malignancy metastatic to the urinary bladder. Anticancer research2014;34:6779-6784. 